\title{
Study of Pressure Distribution in Compliant Contact Wheels for Robotic Surface Finishing
}

\author{
Y.Sun ${ }^{1}$, T.T.Vu ${ }^{1}$ and S.H.Yeo ${ }^{2}$ \\ 1 Rolls-Royce@NTU Corporate Lab, Nanyang Technological University, Singapore \\ 2 School of Mechanical and Aerospace Engineering, Nanyang Technological University, Singapore
}

\begin{abstract}
This paper investigates the compliance characteristics of contact wheels in robotic finishing process, especially pressure distribution - a key parameter for material removal. The current study tackles this problem by developing finite element models of compliant contact wheels to predict pressure distribution with respect to various tool-workpiece scenarios. These models are further verified using pressure film sensors with flat and serrated contact wheels mounted on a robot.
\end{abstract}

\section{Introduction}

Robotic surface finishing processes play a key role in manufacturing industries. Compliant abrasive tools are used extensively, and they are available in a wide variety of forms and shapes [1,2]. This paper focuses on two types of compliant contact wheels, i.e. flat contact wheels and serrated contact wheels, which are shown in Fig. 1 (Left). Unlike hard tools such as grinding wheels which are very stiff, the contact wheels studied in this paper are compliant and their compliance can be controlled by the choice of the stiffness of the rubber in the outer ring and the serration of the serrated contact wheel. Thanks to their compliant nature, these tools improve the surface integrity of the workpiece conforming to the surface profile, and achieving blending.

Existing robotic finishing process relies on Design of Experiment to study empirically various process parameters such as applied force, contact angles, wheel speed, etc. This approach is labour intensive, as well as time consuming and excessive cost required. Therefore, there is a need for a better understanding of the compliancy of contact wheels in robotic finishing process, especially pressure distribution - a key parameter for material removal.

Related works pertaining compliance effects of abrasive tools on the pressure distribution and material removal can be referred from the studies by Vlassak [3] and Umer et. al.[4]. In the first study, a compliant polishing pad removed material through abrasive slurry applied between the pad and the workpiece surface following a method known as chemical mechanical polishing (CMP), whereby the pressure distribution was assumed to follow Hertzian contact model [5]. On the other hand, the latter study employed finite element model to determine the pressure distribution caused by a compliant disc for prediction of surface evolution in robotic polishing. The model was subsequently validated using pressure film sensors.
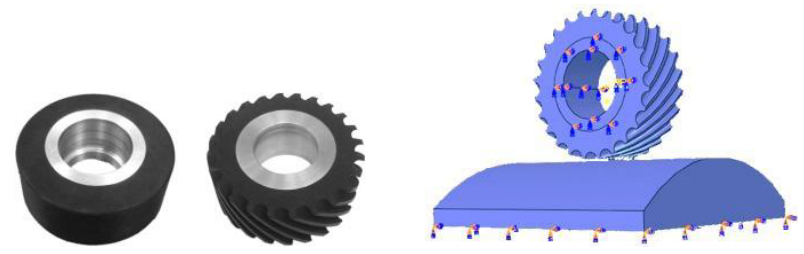

Figure 1. Flat- and serrated contact wheels (Left) and Model setup for serrated contact wheel w.r.t. convex surface (Right).

This paper studies the development of finite element models of compliant contact wheels to predict the pressure distribution with respect to various work surfaces. These models are subsequently verified empirically using pressure film sensors. In addition, this paper compares the established finite element methods with the pressure distribution model based on Hertzian contact theory, hence providing insights into the relationship between industrial application and theoretical models with respect to compliant contact wheels. Pressure measurements are simplified, in that, the pressure distributions are determined without the use of an abrasive belt. These approximated pressure distributions offer a promising way to better understand the mechanism of material removal.

\section{Hertzian Model}

This section presents Hertzian contact theory, which focuses on non-adhesive contact of two elastic solids. A contact is Hertzian if it satisfies the following assumptions [5]: 
1) the surfaces are continuous and non-conforming;

2) the strains are small and within the elastic limit;

3) the surfaces can be considered as half-space and can be locally described by orthogonal radii of curvature;

4) the surfaces are frictionless and non-adhesive.

The contact area is an ellipse and the contact pressure distribution is an ellipsoid.

$$
\left(\frac{x}{a}\right)^{2}+\left(\frac{y}{b}\right)^{2} \leq 1
$$

and

$$
p(x, y)=-p_{0} \sqrt{1-\left(\frac{x}{a}\right)^{2}-\left(\frac{y}{b}\right)^{2}}
$$

where $p_{0}$ is the maximum contact pressure and $a, b$ are the major and minor semi-axes respectively. The details in the calculation of $a, b$ and $p_{0}$ can be found in [5]. If the contact bodies are two parallel cylinders, the contact area is a rectangle with the width $c$ and the length $L$ [5], where $L$ is the length of contact along axis of cylinders. The pressure distribution is

$$
p(x)=\frac{2 F}{\pi a L} \sqrt{1-\frac{x^{2}}{a^{2}}}
$$

where $F$ is the normal polishing force. Due to the complexity in real applications, many contact problems are non-Hertzian, such as those associated with serrated contact wheels because of the discontinuity of the contact body.

\section{Finite Element Models}

This section establishes finite element models for flat contact wheels and serrated contact wheels with respect to workpieces of different geometries.

\subsection{Model Setup}

Each contact scenario is simulated with two contact bodies, including a contact wheel (either flat or serrated), and a workpiece (flat, convex or concave surface).

Similar to real-life conditions, the bottom of the workpiece is fixated, and the hub of the contact wheel is limited to vertical displacement only. A vertical load of $500 \mathrm{~N}$ is assigned and uniformly distributed across the lower half of the centre bore of the wheel hub. Fig. 1 (Right) shows the example of the model setup for contact scenario of serrated wheel, where the reference point is the centre of the contact wheel. The wheel models are meshed with three dimensional, eight node linear hexahedral (C3D8R) elements and ten node quadratic tetrahedron (C3D10) elements respectively. In this paper, the models with flat/concave workpieces are developed by using ABAQUS standard implicit analysis mode while the models with convex workpieces are using ABAQUS explicit analysis mode [6].

The compliance of the contact wheels is characterized by their rubber cushions. The material properties of rubber such as density, Young's Modulus and Poisson's Ratio are $1100 \mathrm{~kg} / \mathrm{m}^{3}, 4.5 \mathrm{MPa}$ and 0.48 respectively. The corresponding material properties of aluminum which constitutes the inner hub of the contact wheel as well as the workpiece can be found in [7]. To capture the contact between the contact wheels and the workpiece, ABAQUS' surface to surface contact algorithm is used for flat contact wheel while ABAQUS' general contact algorithm is used for serrated contact wheel.

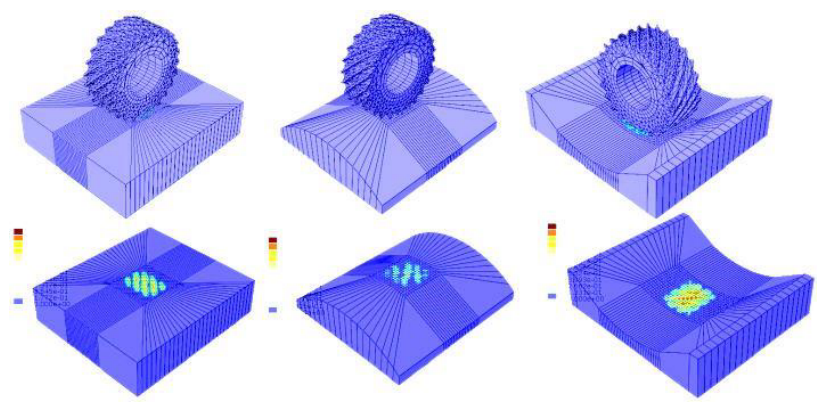

Figure 2. Pressure distribution for serrated contact wheel w.r.t.flat-, convex- and concave surfaces.

\subsection{Simulation Results}

The simulation results for serrated contact wheel with respect to flat, convex and concave surfaces are shown in Fig. 2. Similarly, the simulation results for flat contact wheel with respect to flat, convex and concave surfaces are presented in section 5 . To achieve accurate simulation results within the reasonable computational time, a finer meshing is applied to the area around the vicinity of the contact patch, while a coarser meshing is applied to other areas away from the patch. These finite element models are subsequently validated through comparison with the Hertzian model (section 2) and empirical findings (section 4).

\section{Experiment}

\subsection{Methodology and Setup}

Fujifilm's Prescale Measurement Films are used to measure the pressure distributions over a contact area. In a general setup, the pressure sensor film is placed between a tool and a workpiece of interest. The tool, mounted on a six-axis ABB robot, is vertically pressed down against the workpiece until a force of $500 \mathrm{~N}$ is registered by an ATI force sensor. The experimental setup is shown in Fig. 3.

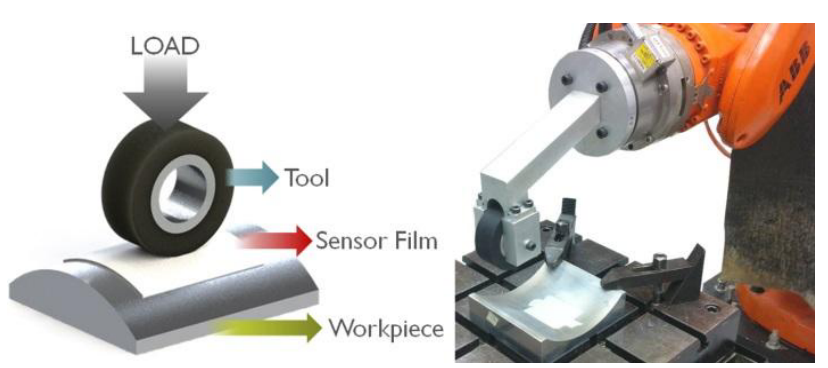

Figure 3. Workpiece covered with Fujifilm's Prescale Measurement Film (Left), ABB robot mounted with flat contact wheel (Right). 
The pressure-exposed film is scanned and analyzed using a specialized post-process interpretive system: the FPD-8010E, which produces the pressure values pixel by pixel. To fully capture the pressure spectrum of interest, three types of pressure films (LW, LLW and LLLW) with the combined measurement range from 0.2 to $10 \mathrm{MPa}$ are used [8].

\subsection{Results}

In a representative case, Table 1 depicts the contact patch and pressure distribution created by the contact between serrated contact wheel on convex surface, which are measured respectively by LW, LLW and LLLW Prescale pressure films. LLW film, which displays the largest area of pressure in its measurable range, apparently captured the majority of pressure magnitudes.

Table 1. Pressure distribution recorded by 3 film types.

\begin{tabular}{|c|c|c|}
\hline \multicolumn{3}{|c|}{ Sensor Film and Pressure Range (Mpa) } \\
\hline LW & LLW & LLLW \\
\hline $2.5-10$ & $0.5-2.5$ & $0.2-0.5$ \\
\hline & & \\
&
\end{tabular}

* $\quad, \quad$ and $\square$ are respectively corresponding to pressure values that are under, within and above the measurable range of the sensor film.

On the other hands, LLLW film is the most sensitive among the three film types, making it the optimal indicator for the shape and dimension of the contact patch. Table 2 depicts different contact patches and pressure distribution patterns recorded by LLLW film.

Table 2. Pressure distribution in 6 contact scenarios.

\begin{tabular}{|c|c|c|c|}
\hline Surface & Flat & Convex & Concave \\
\hline \multirow{2}{*}{ Flat Wheel } & & & \\
& & & \\
\hline Serrated Wheel & & & \\
& & & \\
\hline
\end{tabular}

\section{Discussion}

\subsection{Methodology}

The pressure distribution results collected from Hertzian models, finite element models and experiments are juxtaposed into a common platform, where the pressure values are plotted in the same three dimensional graph (Fig. 4). The origin of the graph is located at the centre of the contact patch. Here, the pressure magnitude in the direction vertical to the work-piece is set as z-axis, and the plane which is perpendicular to z-axis is $x-y$ plane. It can be seen that Fig. 4 includes six pictures. For each of the picture, the top left sub-picture shows the contact patch in $x-y$ plane, the top right sub-picture shows the pressure distribution $(\mathrm{MPa})$ against $\mathrm{x}-\mathrm{y}$ plane, the bottom left and right sub-pictures show the projections of the top right graph along $\mathrm{x}$-direction and $\mathrm{y}$-direction respectively.

Due to the complex geometry of serrated contact wheel which results in non-Hertzian contact problem, only contact scenarios associated with flat contact wheel are presented using Hertzian formulae. As such, for the pressure distribution by flat contact wheel, finite element models are compared with experiment results and Hertizian model simultaneously (Fig. 4a-c). For the pressure distribution with serrated contact wheel, only finite element models and experiment results are compared. (Fig. 4d-f).

\subsection{Model Accuracy}

The pressure distribution is characterized by the area of the contact patch and the magnitude of the pressure profile.

\subsubsection{Pressure distribution shape}

In general, coherent results are observed in pressure results for flat wheel on flat/convex/concave surfaces and serrated contact wheel on flat/convex/concave surfaces.

More specifically, elliptical contact patch is consistently observed in the Hertzian-, finite element- and experimental results for the contact scenario between flat contact wheel and convex surface (Fig. 4b). Similarly, rectangular contact patch is found in all the results for contact of flat wheel on flat and concave surface (Fig 4a and Fig. 4c).

On the other hands, contact patches observed from experiments with serrated contact wheels (Fig. 4d-f) relatively matched those simulated from finite element models. In all contact scenarios, dimensional differences are observed across the contact patches from Hertzian models, finite element models and experiments. For instances, the contact patch areas generated via finite element models appeared proportionally larger than those measured empirically. The dimensional difference may stem from the presence of friction in the experiments, which is assumed to be negligible for simulation simplicity.

\subsubsection{Pressure distribution value}

In general, the overall pressure profiles appears consistent across results from Hertzian models, finite element models and experiments.

High pressure concentration is observed at the ends of the pressure profile generated from experiment results, as shown in Fig. 4a and Fig.4c. 


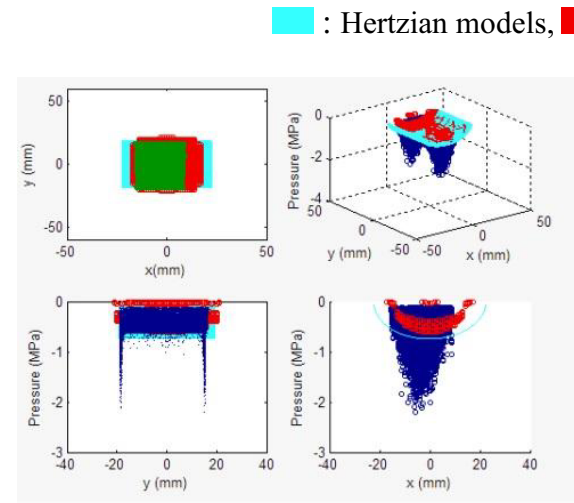

(a)

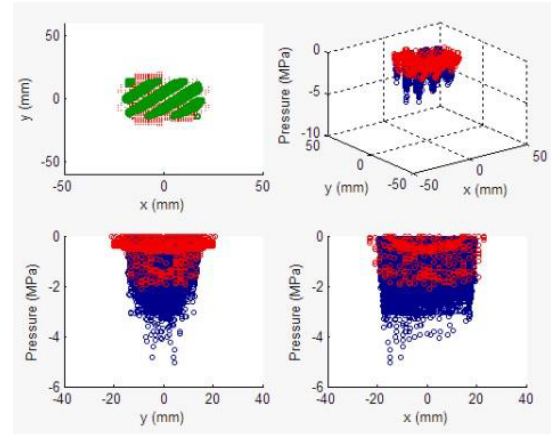

(d)

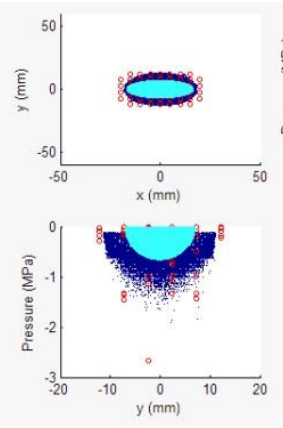

(b)

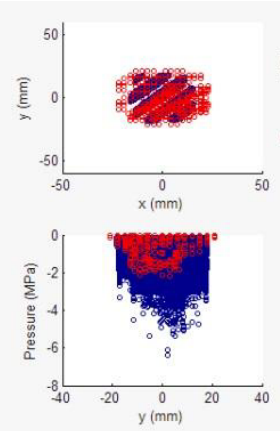

(e) : experiments, : overlapping plots.

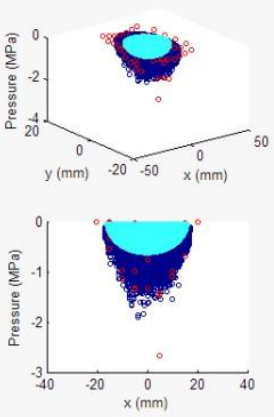

(b)

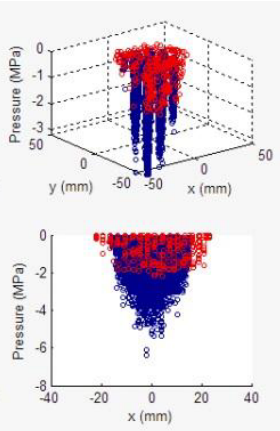

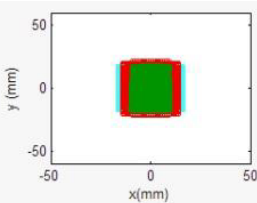
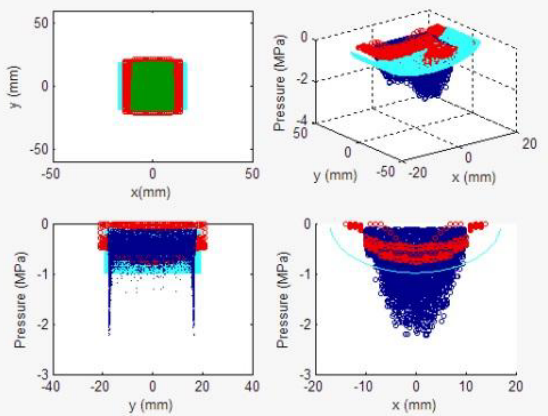

(c)
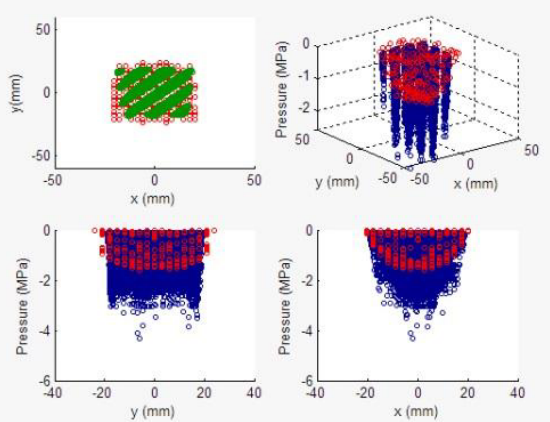

(f)

Figure 4. (a to c) Flat contact wheel w.r.t. flat-, convex- and concave workpieces \& (c to f) Serrated contact wheel w.r.t. flat-, convexand concave workpieces.

The high pressure is associated with discontinuity at the circumferential edge of the contact wheel. This is not reflected in both Hertzian and finite element models, the former is due to the assumption of infinitive contact cylinders while the latter is due to simplification of complex material deformation at the edges of wheel. The aforementioned high pressure also causes relatively narrower pressurized area in empirical results.

Similarly, high pressure is expected at the edge of each tooth in the serrated wheel (due to discontinuity). This explains the higher pressure values from experiment results as compared to Hertzian and finite element models, despite the similarity in the profile shapes (Fig. 4d-f). Moreover, differences in pressure values across Hertzian models, finite element models and experiments may also result from simulation and empirical errors, such as those associated with estimated material data, mesh sensitivity, force calibration and the sensitivity of the pressure film.

\section{Conclusion}

To determine the pressure distribution caused by compliant abrasive tools, finite element models for flatand serrated contact wheels were presented. Different contact scenarios with representative flat, convex and concave workpieces were covered. The finite element models were subsequently verified by comparison with theoretical Hertzian model and empirical findings. The results across three methods show consistency in the shape of the contact patch and the pressure profile.
Differences in the pressure values are associated with discontinuity at the edge of tools. Further improvement in simulation inputs and empirical set-ups are necessary to increase accuracy of the model development and verification. The results have laid a foundation for future work in predicting material removals using compliant abrasive tools.

\section{Acknowledgement}

This work was conducted within the Rolls-Royce@NTU Corporate Laboratory with support from the National Research Foundation (NRF) Singapore under the Corp Lab@University Scheme. The authors would like to acknowledge the support provided by Arthur Wee and Sudhan R. Gopikrishnan.

\section{References}

1. M. J. Jackson, and J. P. Davim: Machining with abrasives, Springer (2011).

2. A. Arunachalam and S. Idapalapati and S. Subbiah: Multi-criteria decision making techniques for compliant polishing tool selection, Int. J. Adv. Manuf. Technol, 79, pp.519-530, Springer (2015).

3. Vlassak, J. J.: A model for chemical-mechanical polishing of a material surface based on contact mechanics, J. Mech. Phys. Solids, 52, pp 847-873 (2004). 
4. M. Umer, K. Saptaji, and S. Subbiah, Study of pressure distribution in compliant coated abrasive tools for robotic polishing, International Manufacturing Science and Engineering Conference, pp. 855-860 (2012).

5. K. L. Johnson, Normal contact of elastic solids Hertz theory, in Contact Mechanics, K. L. Johnson, Ed., ed: Cambridge University Press, pp. 84-106 (1987).

6. Dassault Systèmes, Abaqus 6.14 Documentation (2014).

7. I. Marinescu, W. Ioan, W. Brian, B. Dimitrov, Boris and I. Inaski: Tribology of abrasive machining processes, Elsevier (2004).

8. Fujifilm, Fuji Prescale Film Product Guide (2011). 